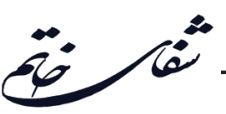

\title{
Experiences of Living with Neurofibromatosis in A Young Woman: A Case Report
}

Mona Rezapoor Esfahani ${ }^{1}$, Leila Jouybari ${ }^{2 *}$, Akram Sanagoo ${ }^{2}$, Fereshte Araghian Mojarad ${ }^{1}$

'Student Research Committee, Golestan University of Medical Sciences, Gorgan, Iran

${ }^{2}$ Nursing Research Center, Goletsan University of Medical Sciences, Gorgan, Iran

\section{ABSTRACT}

Introduction: Neurofibromatosis is a rare autosomal dominant genetic disease causing range of symptoms, especially skin lesions, and seriously affects the patients' quality of life. The aim of this study was to describe the experiences of living with neurofibromatosis type 1 (NF1). Case Description: This qualitative case report examined the experiences of living with NF1 in a 25-year-old woman. The data were collected through interactive semistructured interviews and analyzed by Van Manen phenomenological approach. Strategies to ensure the rigor of study were prolonged engagement, triangulation, and member checking. Results: Data analysis revealed two main issues, including "fear of the unknown", and "try to cope with". Hiding, dependent on my family, joining social networks, and hope for a cure were other important issues for this patient. Conclusion: Family and social support groups could help the patients in dealing with loneliness, fear and anxiety, and find herself in caring climate with mutual understanding and empathetic connection.

Key words:

1. Neurofibromatosis 1

2. Genetic Diseases, Inborn 3. Iran

*Corresponding Author: Leila Jouybari

E-mail:jouybari@goums.ac.ir 
تجارب زندكى با نوروفيبروماتوز در يك زن جوان: گزارش موردى

\author{
مونا رضايور اصفهانى'، ليلا جويبارى זّ، اكرم ثناتوّ، فرشته عراقيان مجرد' \\ 'كميتهُ تحقيقات دانشجويى، دانشَاه علوم يزشكى كلستان، كر كان، ايران

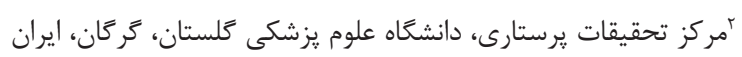

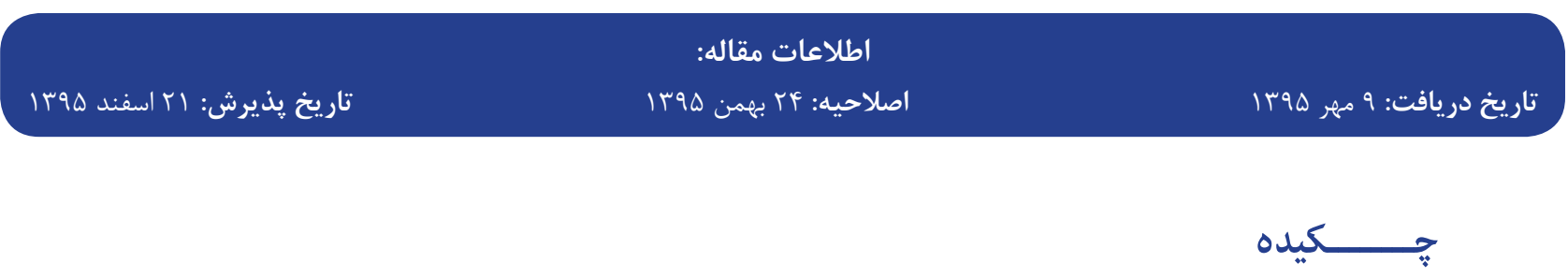

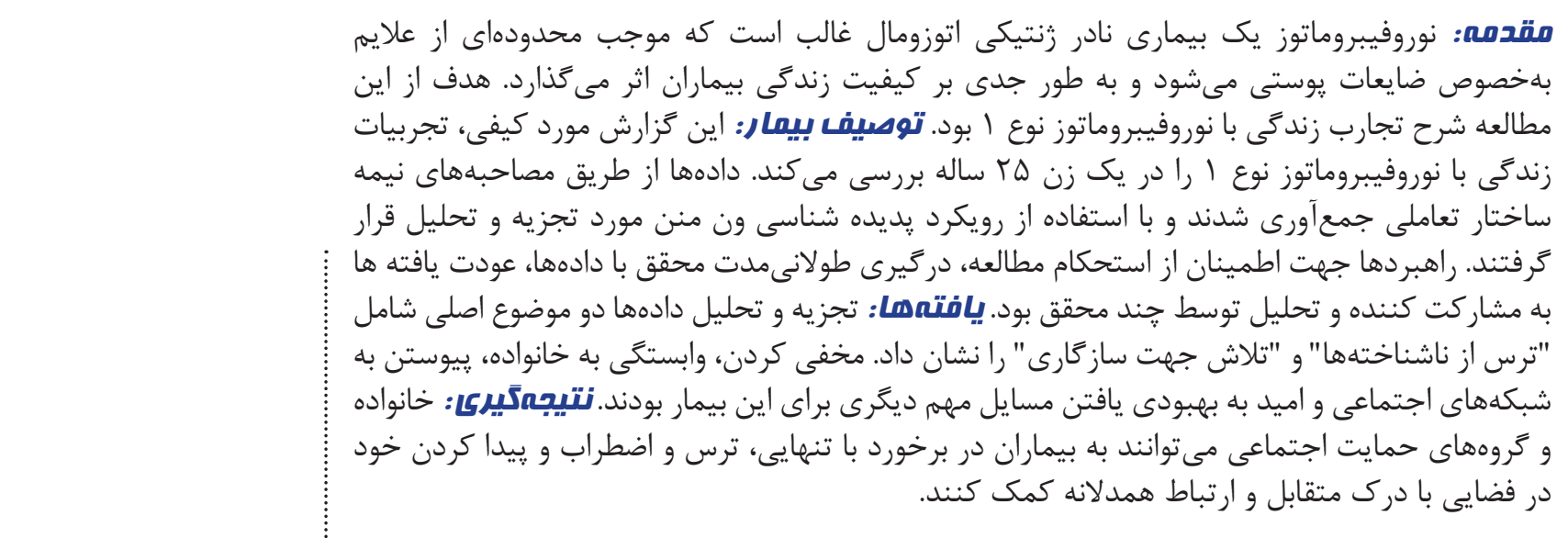

كليد وازهها:

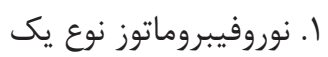

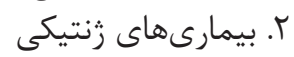
در حال تولد بران r. ايران

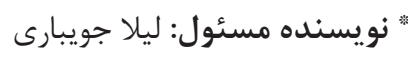
آدرس الكترونيكى: jouybari@goums.ac.ir 


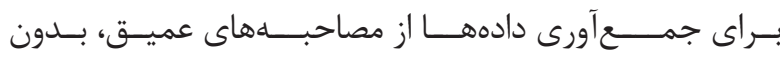

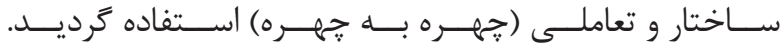

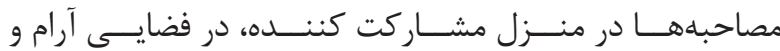

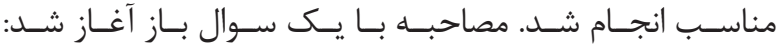

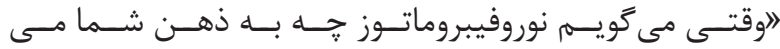

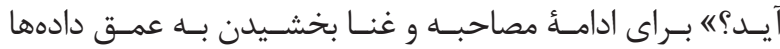

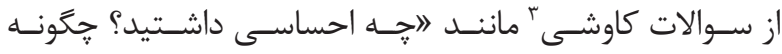

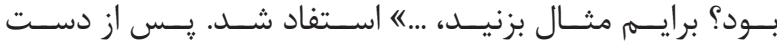

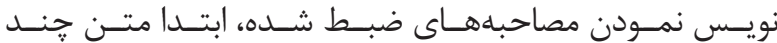

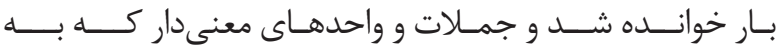

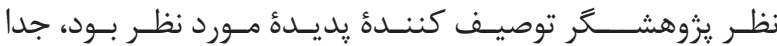

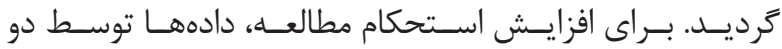

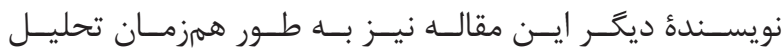

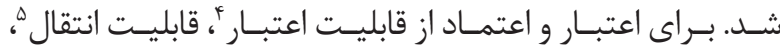

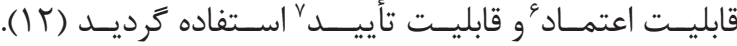

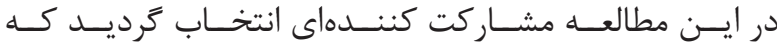

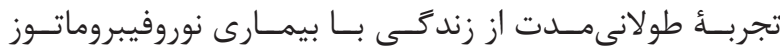

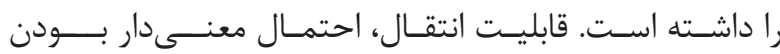

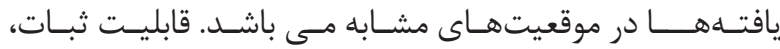

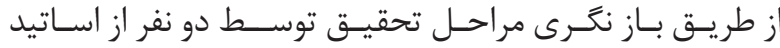

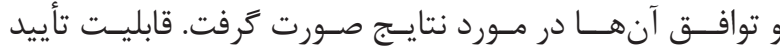

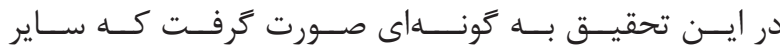

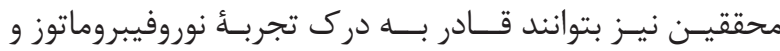

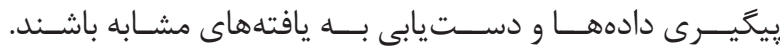

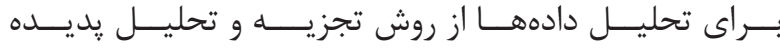

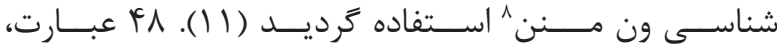

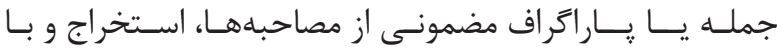

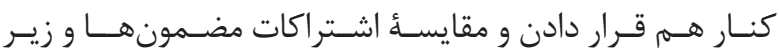

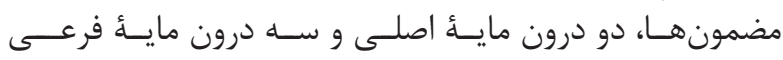

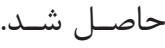

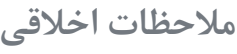

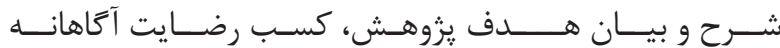

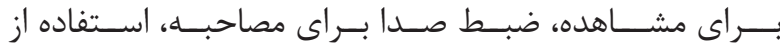

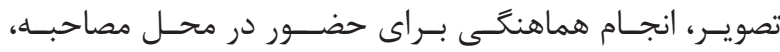

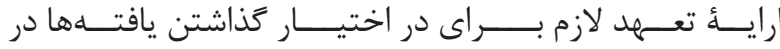

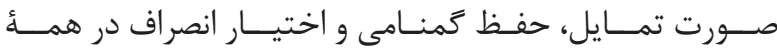

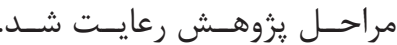

كَزارش مورد

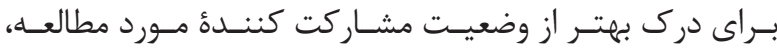

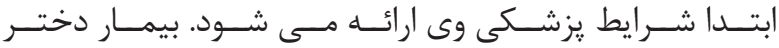

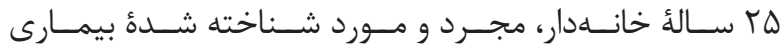

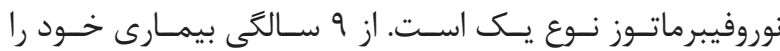

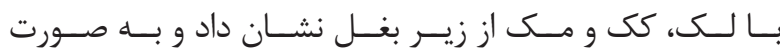

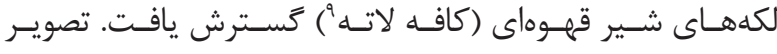

${ }^{1}$ Von Recklinghausen

${ }^{2}$ National institute health

${ }^{3}$ Probing

${ }^{4}$ Credibility

${ }^{5}$ Transferability

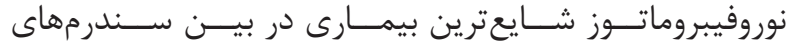

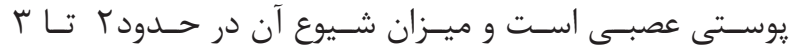

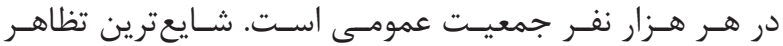

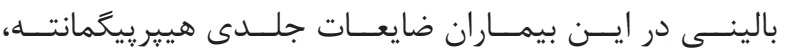

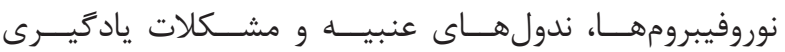

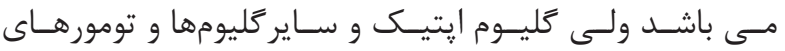

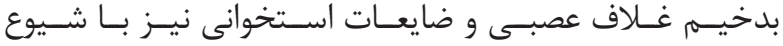

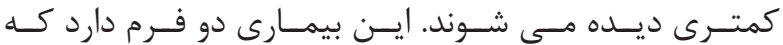

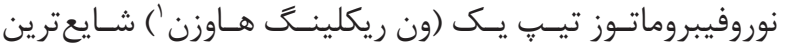

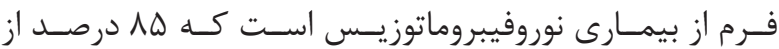

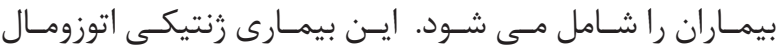

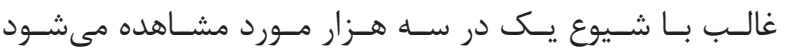

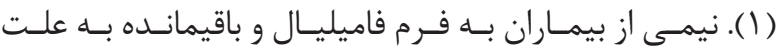

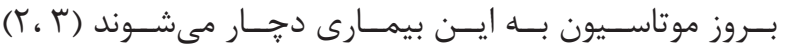

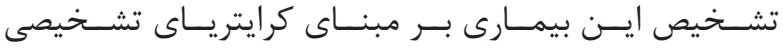

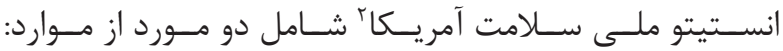

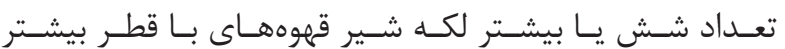

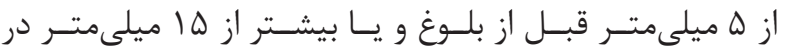

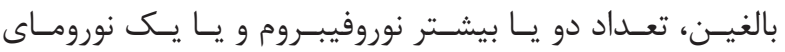

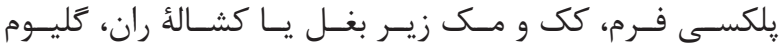

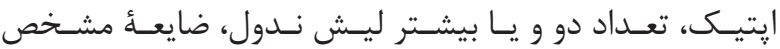

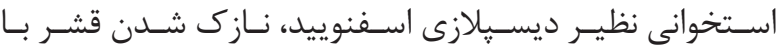

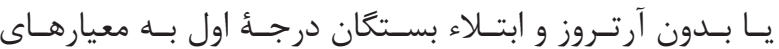

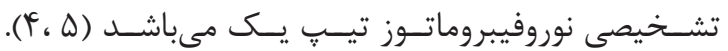

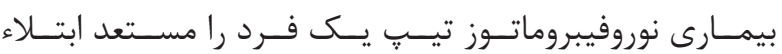

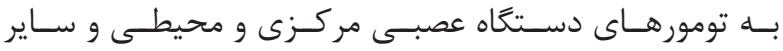

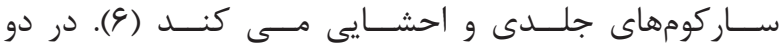

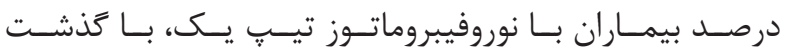

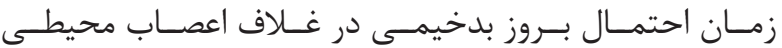

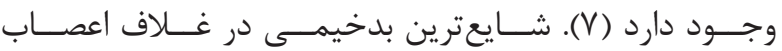

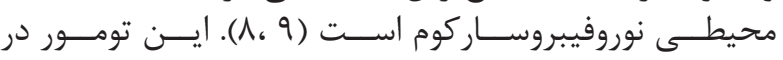

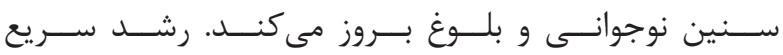

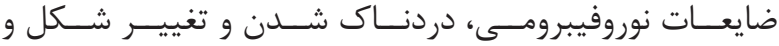

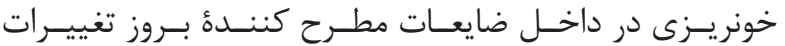

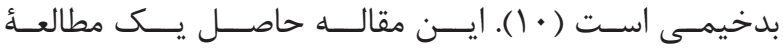

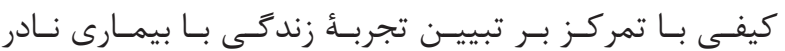

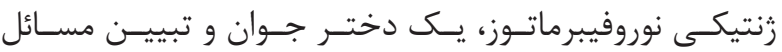

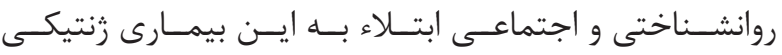

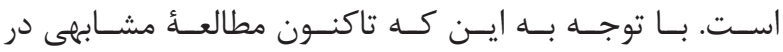

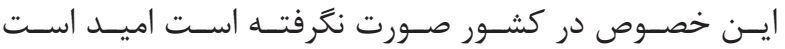

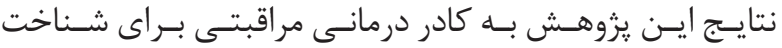

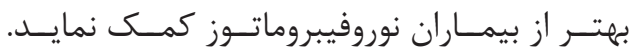

\section{توصيف بيمار}

مطالعــهُ حاضـر يـك حَـزارش مــوردى بــا روش كيفـى اسـت.

${ }^{6}$ Dependability

${ }^{7}$ Conformability

${ }^{8}$ Max van manen

${ }^{9}$ Café au lait spot 


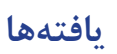

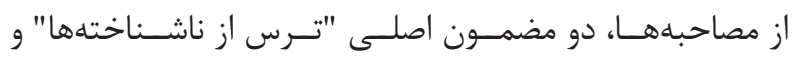

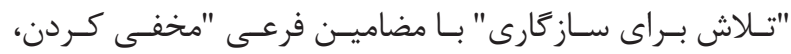

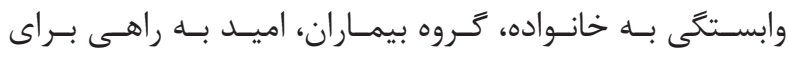

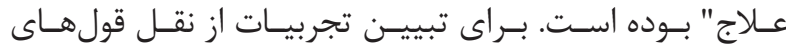

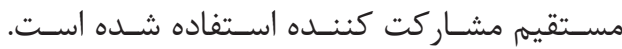

ترس از ناشناختهها

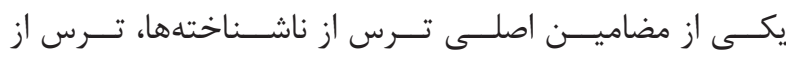

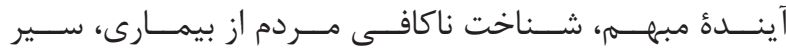

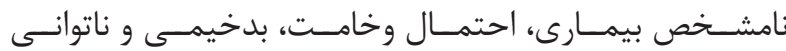

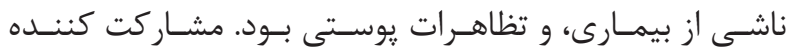

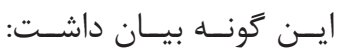
الاصـلا يكـى از ترسـهام هميـن آينــداى هسـت كـهـ نمى دونسم

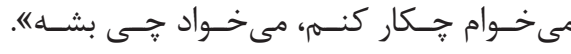

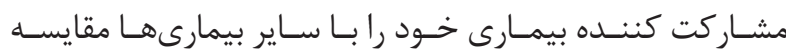

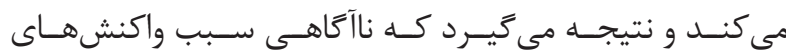

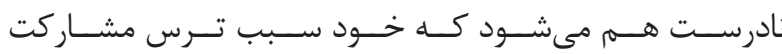

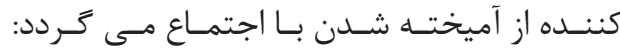

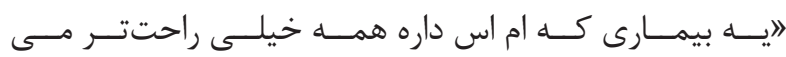

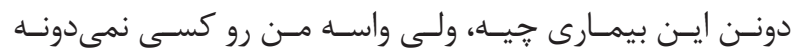

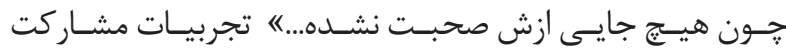

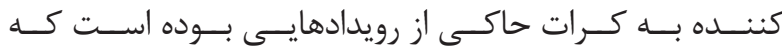

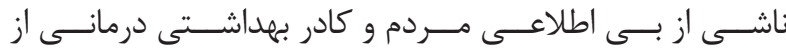

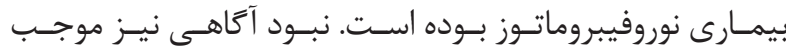

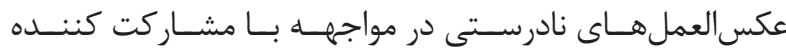

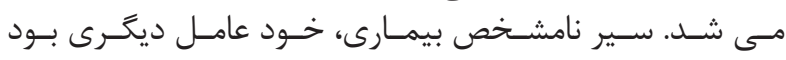

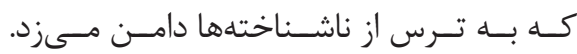

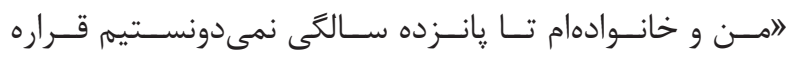

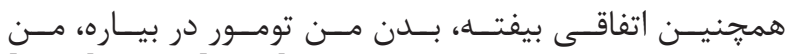

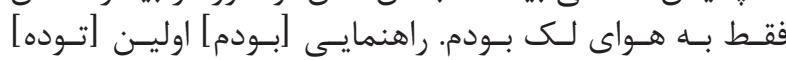

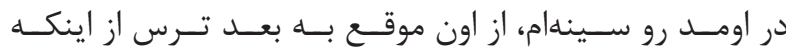

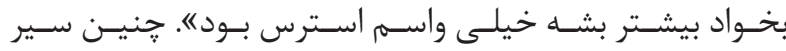

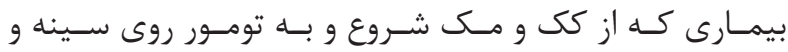

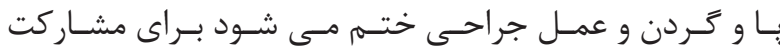

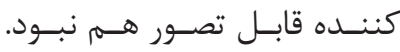

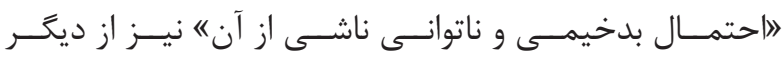

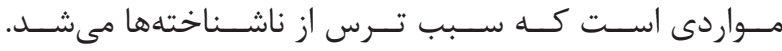

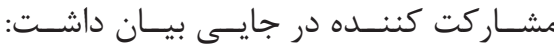

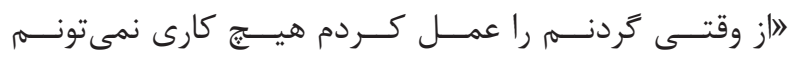

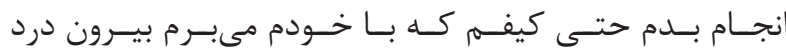

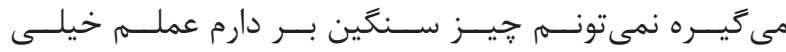

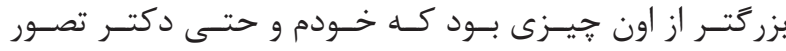

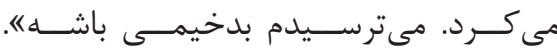

${ }^{10}$ Magnetic resonance imaging

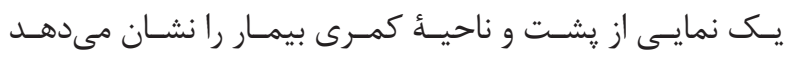

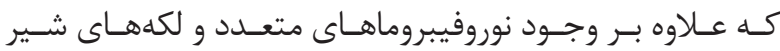

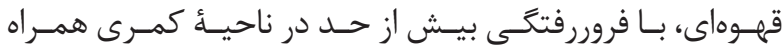

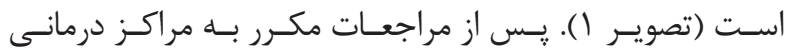

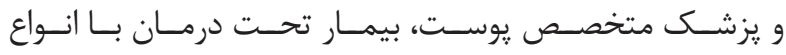

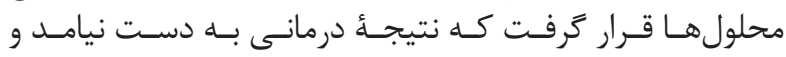

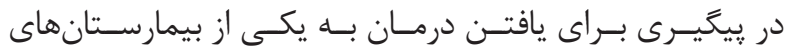

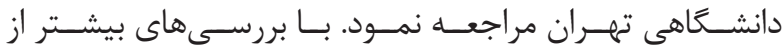

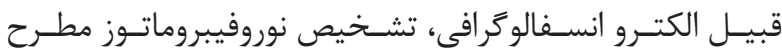

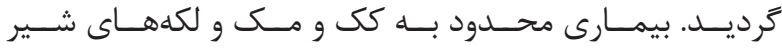

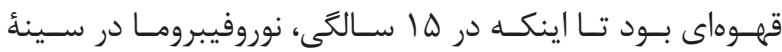

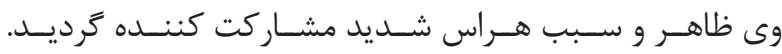

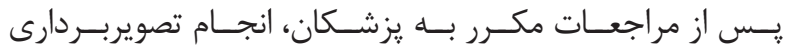

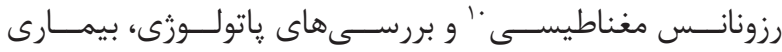

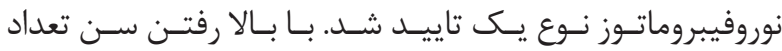

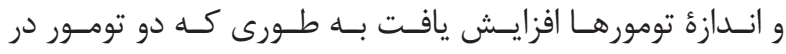

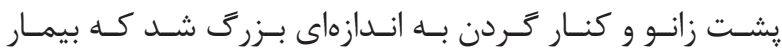

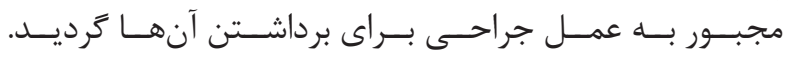

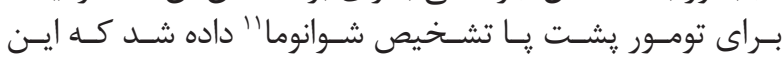

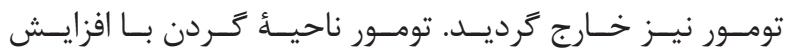

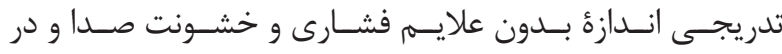

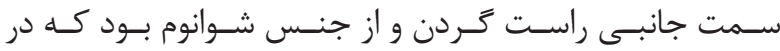

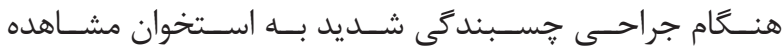

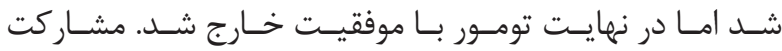

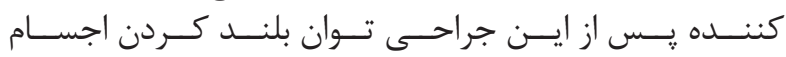

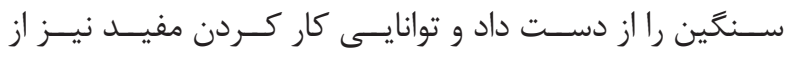

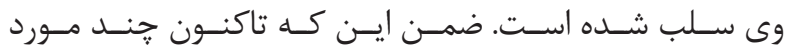

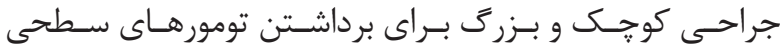

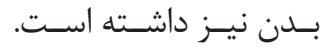

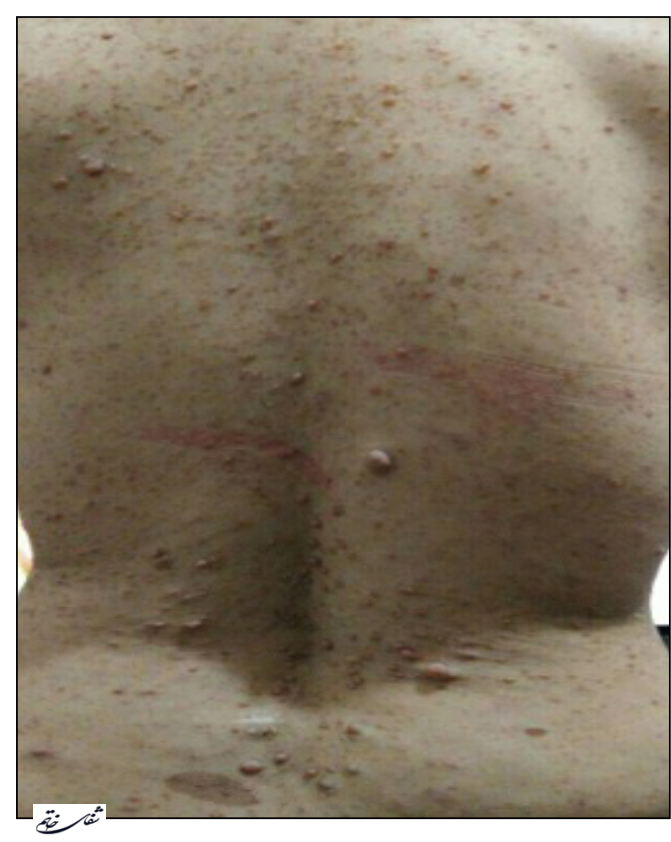

تصوير ا- نمايى از لكههاى شير قهوهاى، نورفيبروماهاى متعدد يوستى و فرو

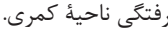




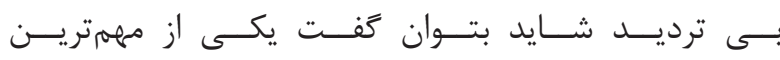

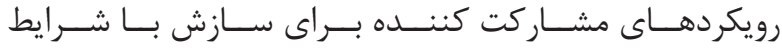

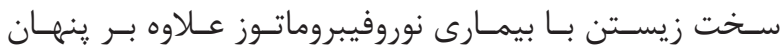

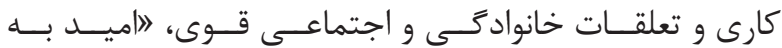

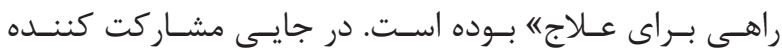
اظظهـار داشــت

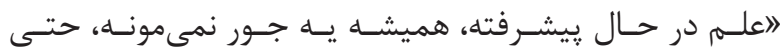

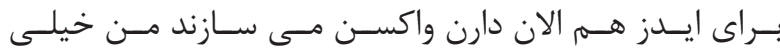

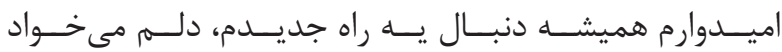

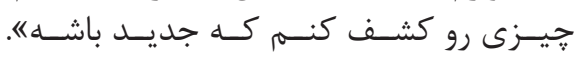

\section{بحث و نتيجه}

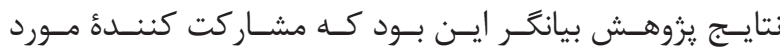

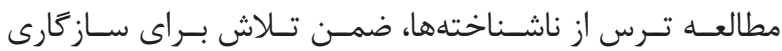

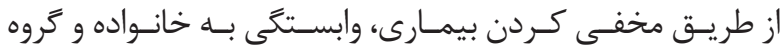

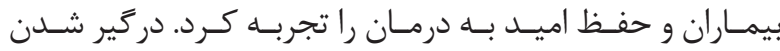

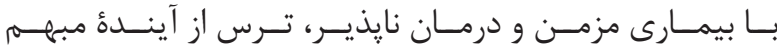

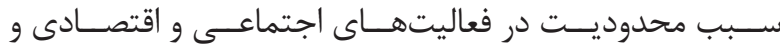

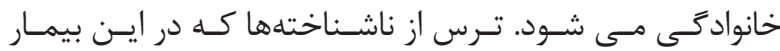

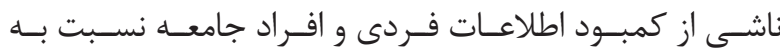

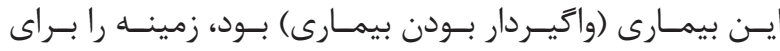

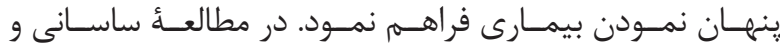

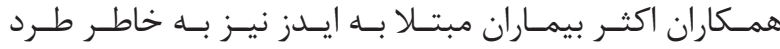

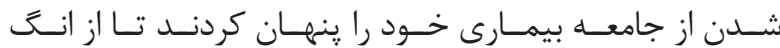

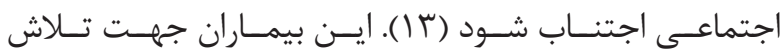

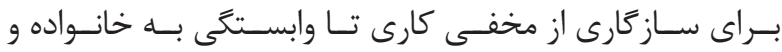

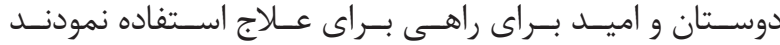

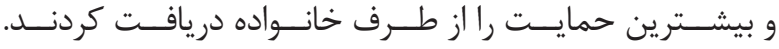

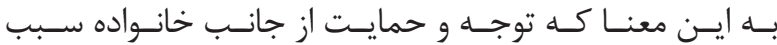

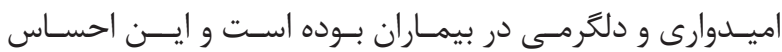

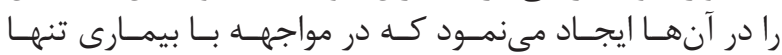

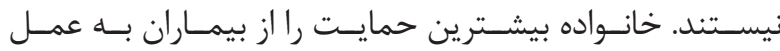

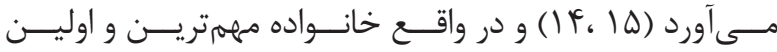

منبـع حمايتـى اسـت (1) (1) (1) (1)

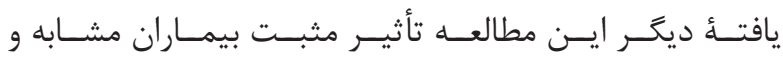

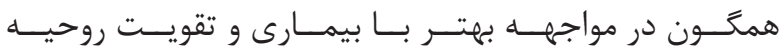

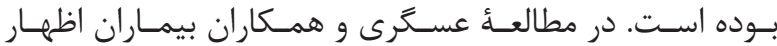

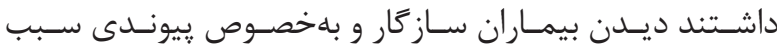

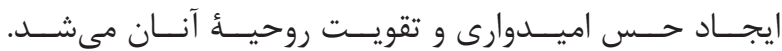

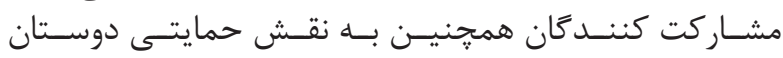

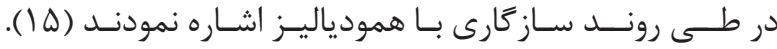

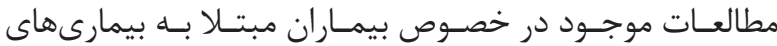

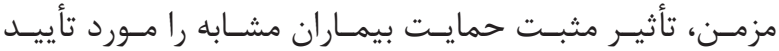

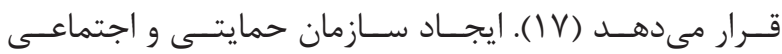

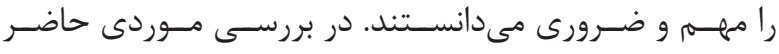

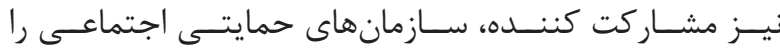

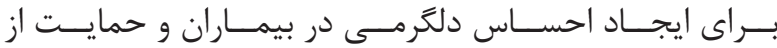

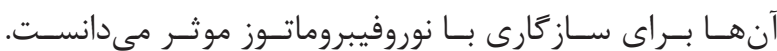

تلاش براى سازگًارى

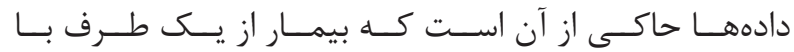

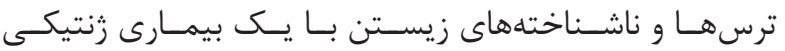

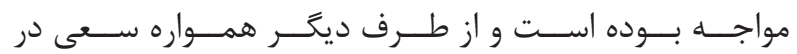

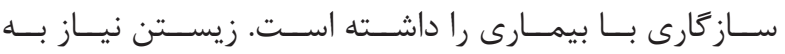

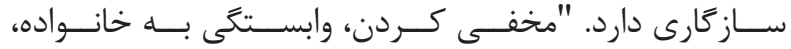

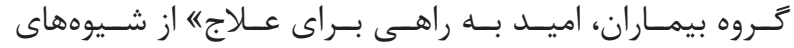

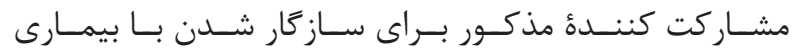

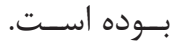

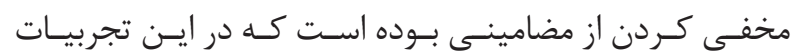

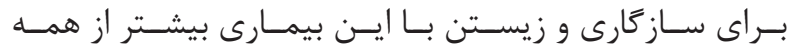

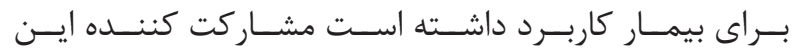

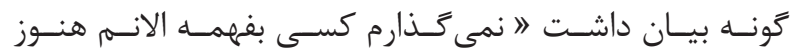

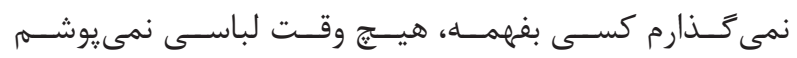

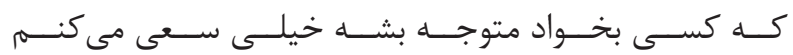
مخفى كنــم ايسن موضـوع رو از همــهـه

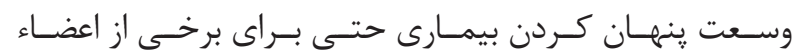

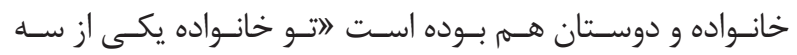

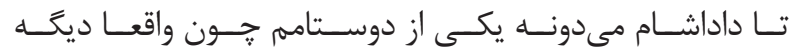

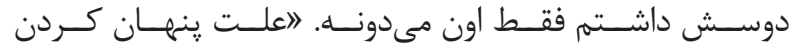

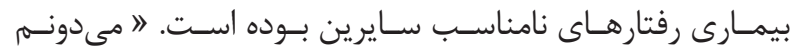

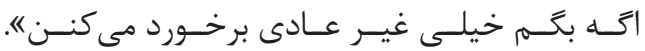

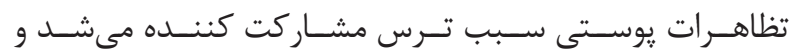

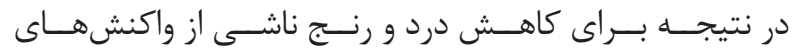

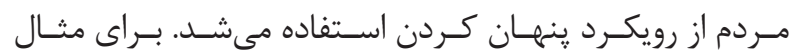

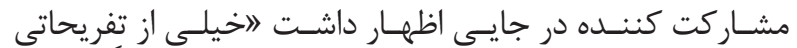

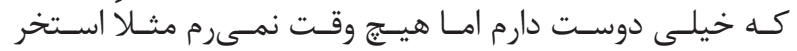

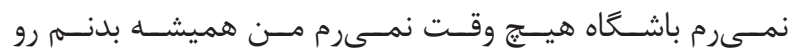

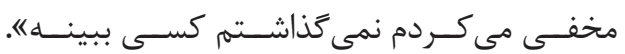

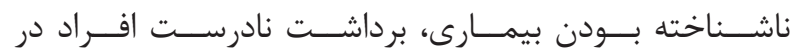

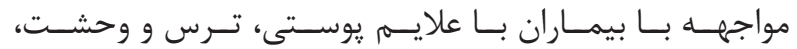

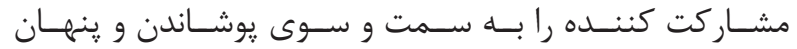

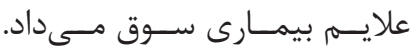

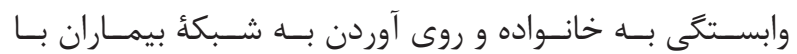

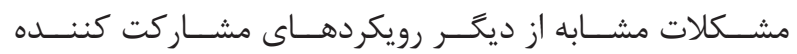

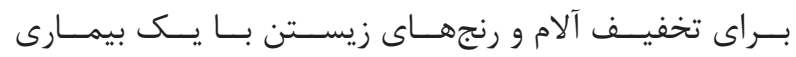

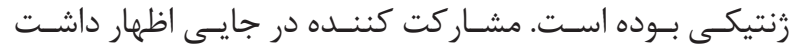

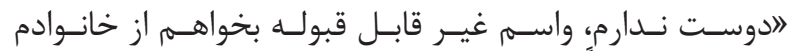

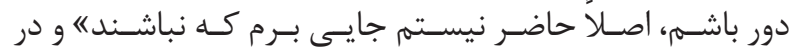

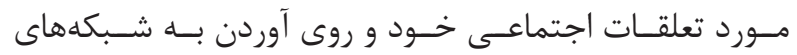

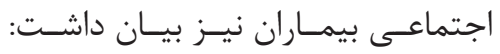

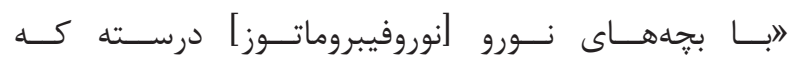

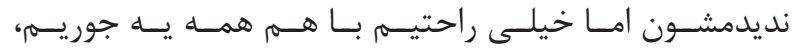

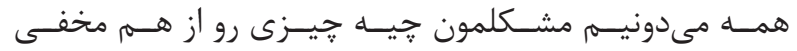

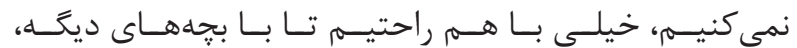

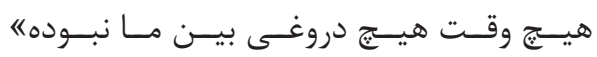




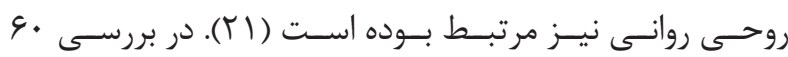

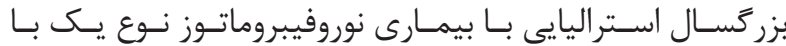

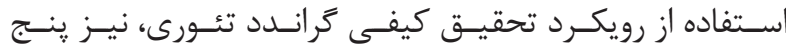

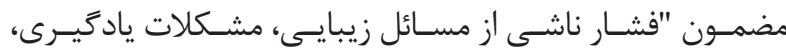

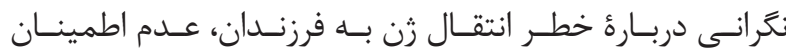

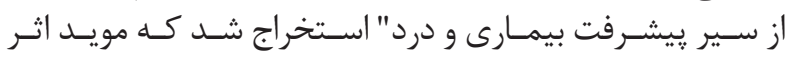

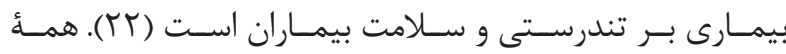

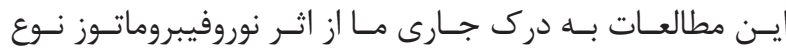

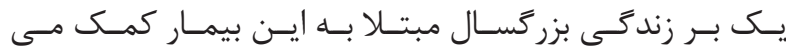

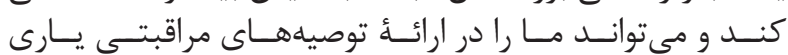
نمايـد.

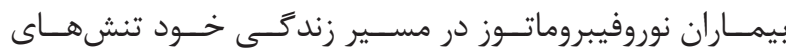

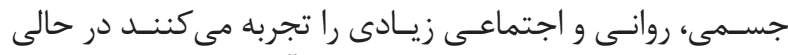

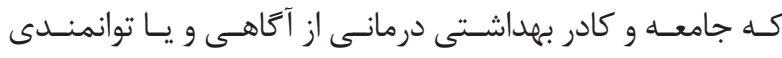

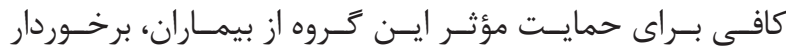

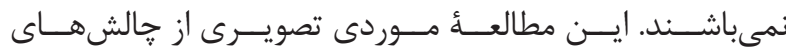

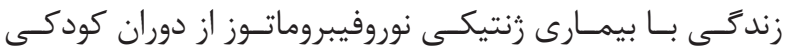

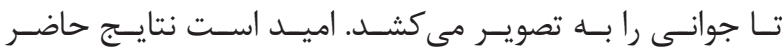

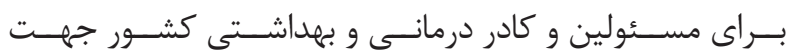

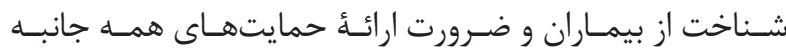

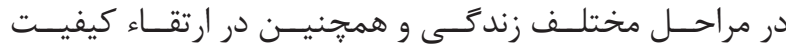

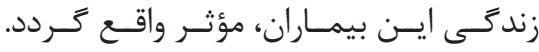

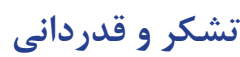

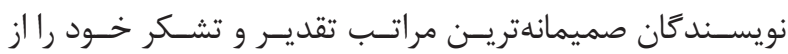

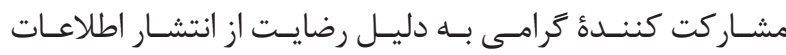

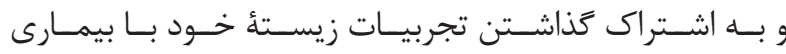

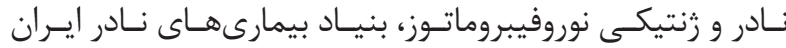

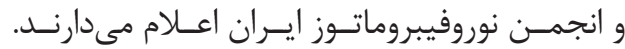

1. Lammert M, Friedman J, Kluwe L, Mautner V. Prevalence of neurofibromatosis 1 in German children at elementary school enrollment. Arch Dermatol. 2005; 141: 71 .

2. Riccardi V. Neurofibromatosis: phenotype, natural history, and pathogenesis. Baltimore: Johns Hopkins University Press. 1992.

3. North K. Neurofibromatosis type 1: review of the first 200 patients in an Australian clinic. J child Neurol 1993; 8: 395 .

4. DeBella K, Szudek J, Friedman J. Use of the national institutes of health criteria for diagnosis of neurofibromatosis 1 in children. Pediatrics 2000; 105: 608 .

5. Gutmann DH, Aylsworth A, Carey JC, Korf B, Marks $\mathrm{J}$, Pyeritz RE, et al. The diagnostic evaluation and

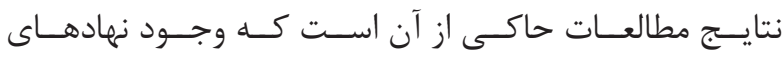

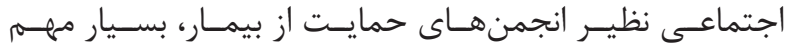

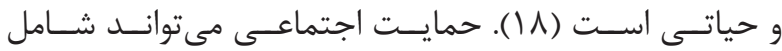

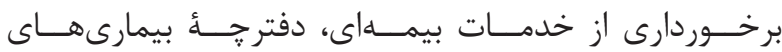

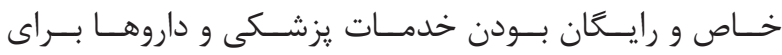

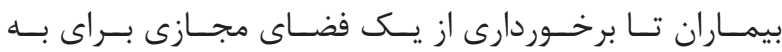

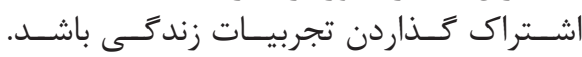

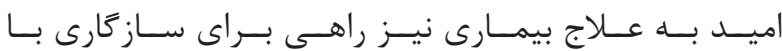

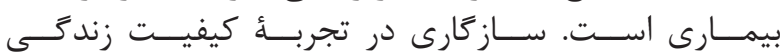

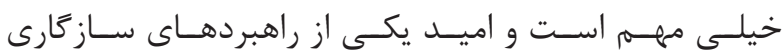

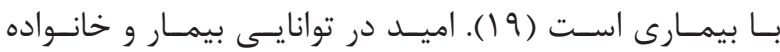

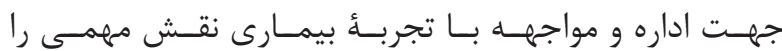

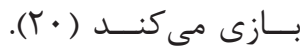

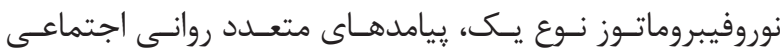

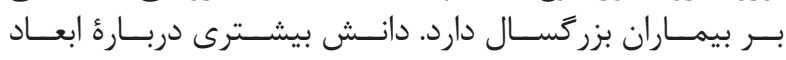

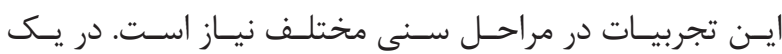

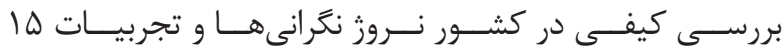

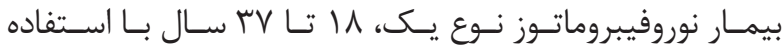

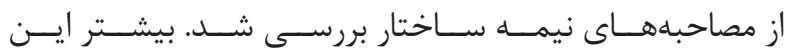

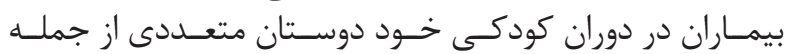

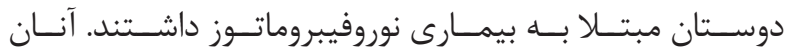

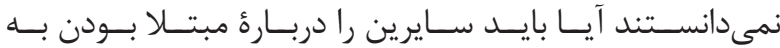

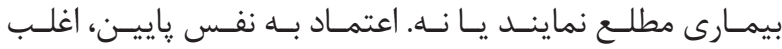

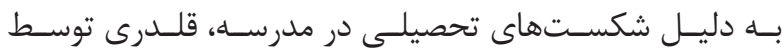

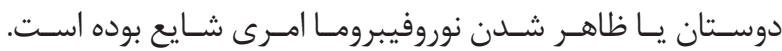

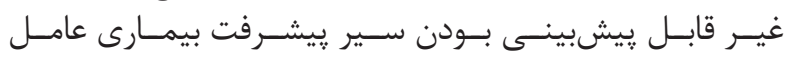

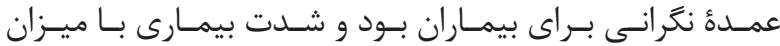

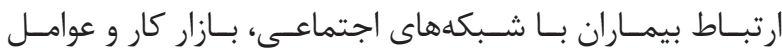

منابع

multidisciplinary management of neurofibromatosis 1 and neurofibromatosis 2. JAMA. 1997; 278: 51.

6. Colman S, Williams C, Wallace M. Benign neurofibromas in type 1 neurofibromatosis (NF1) show somatic deletions of the NF1 gene. Nat Genet. 1995; 11(1): 90-2.

7. Ducatman BS, Scheithauer BW, Piepgras DG, Reiman HM, Ilstrup DM. Malignant peripheral nerve sheath tumors. a clinicopathologic study of 120 cases. Cancer. 1986; 57(10): 2006-21.

8. Plon S, Riccardi V. The phakomatoses and other neurocutaneous syndromes. $3^{\text {rd }}$ ed. Philadelphia: JB Lippincott. 1999.

9. Gutmann D, Collins F. Neurofibromatosis 1. The metabolic and molecular bases of inherited disease, scriver. New York: McGraw-Hill. 2001. 
10. Pauluzzi P, Kokelj F. Segmental neurofibromatosis: a rare or underestimated disease? J Eur Acad Dermatology Venereol. 1997; 9: 169-72.

11. Van MM. Researching lived experience. Human science for an action sensitive pedagogy. Ontario: Althouse Press. 2000.

12. Mac DA. Living with angina pectoris a phenomenological study. EJCNURSE. 2002; 1: 265-72.

13. Sassani L, Naji A, Abedi HT. The experience of social stigma in AIDS patients: a phenomenological study. Quarterly Journal of Sabzevar University of Medical Sciences 2013; 20(4): 487-95.

14. Rustoen T. Hope and quality of life, two central issues for cancer patients: a theoretical analysis. Cancer Nurs. 1995; 18(5): 355-61.

15. Asgari MR, Mohammadi E, Fallahi Khoshknab M, Tamadon M. The perception of chronic renal failure patients from advocacy resources in adjustment with hemodialysis: a qualitative study. Care Critical of Journal Iranian. 2011; 3(4): 133-42.

16. Polaschek N. The experience of living on dialysis: a literature review. Nephrol Nurs J. 2003; 30(3): 303-13.

17. Jouybari L, Oskouie S, Ahmadi F. Comfort of hospitalized patients: a missed concept. Bimonthly Iran J of Nurs. 2006; 19(47): 90-110.

18. Mallik M, McHale J. Support for advocacy. Nurs Times. 1995; 91(4): 28-9.

19. Rezaee N, Rafii F, Mardani M, Ranjbar H. A concept analysis of hope of patient recovery among nurses in intensive care: a hybrid model. J Qual Res Health Sci . 2013; 2(2): 100-10.

20. O'Connor P. Hope: a concept for home care nursing. Home Care Provid. 1996; 1(4): 174-9.

21. Hummelvoll G, Antonsen KM. Young adults' experience of living with neurofibromatosis type 1 . J Genet Couns. 2013; 22(2): 188-99.

22. Crawford HA, Barton B, Wilson MJ, Berman Y, McKelvey-Martin VJ, Morrison PJ. The impact of neurofibromatosis type 1 on the health and wellbeing of Australian adults. J Genet Couns. 2015; 24(6): 931-44. 